\title{
Effect of strain-induced orbital splitting on the magnetic excitations in undoped cuprates
}

\author{
Dheeraj Kumar Singh ${ }^{1}$ and Yunkyu Bang ${ }^{2,3}$ \\ ${ }^{1}$ School of Physics and Materials Science, Thapar Institute of Engineering and Technology, Patiala-147004, Punjab, India \\ ${ }^{2}$ Department of Physics, POSTECH, Pohang 790-784, Korea and \\ ${ }^{3}$ Asia Pacific Center for Theoretical Physics, Pohang, Gyeongbuk 790-784, Korea
}

(Dated: December 4, 2020)

\begin{abstract}
We investigate the magnetic excitations in view of the recent reports suggesting that the spin-wave energy may exhibit a significant dependence on the in-plane strain of a thin film of $\mathrm{La}_{2} \mathrm{CuO}_{4}$. The nature of dependence, as we find, can be explained naturally within a two-orbital model based on the $d_{x^{2}-y^{2}}$ and $d_{3 z^{2}-r^{2}}$ orbitals. In particular, as the orbital-splitting energy between the $d_{x^{2}-y^{2}}$ and $d_{3 z^{2}-r^{2}}$ orbitals increases with compressive strain, the zone-boundary spin-wave energy hardens. However, the hardening persists only until the orbital splitting reaches $\sim 2 \mathrm{eV}$, beyond which there is no significant change. The behavior of zone-boundary spinwave energy is explained in terms of the extent of hybridization between one of the exchange-split $d_{x^{2}-y^{2}}$ band which is nearly half filled and the $d_{3 z^{2}-r^{2}}$ band. The role of second-order antiferromagnetic superexchange process involving the inter-orbital hopping is also discussed.
\end{abstract}

PACS numbers:

\section{INTRODUCTION}

The origin of unconventional superconductivity has been a recurrent theme since the discovery of high- $T_{c}$ cuprates in the late eighties [1-4]. The last decade has witnessed the discovery of another large family of multiband superconductors based on iron, which are also believed widely to be unconventional in nature $[5,6]$. A striking similarity between the two class of superconductors is that a long-range magnetic order is exhibited by the parent compounds that gives way to superconductivity on doping either holes or electrons [7]. Thus, the idea that the unconventional superconductivity may be mediated by the spin fluctuations is strengthened further and therefore the nature of such fluctuations can be the key to the understanding of pairing mechanism.

The spin-wave excitations in the Mott-antiferromagnetic phase of high- $T_{c}$ cuprates show a more dispersive behavior near the zone-boundary in comparison to the Heisenberg antiferromagnet with only nearest-neighbor exchange coupling [8-12]. The deviation was explained by incorporating the exchange couplings beyond the nearest neighbor in the Heisenberg model or by considering hopping beyond the nearest neighbor in the one-orbital Hubbard model [1315]. Recent experiments based mainly on the resonant inelastic x-ray spectroscopy (RIXS) have unfolded several new features which are difficult to explain within the one-orbital model $[16,17]$. One such remarkable feature is that the spinwave energy exhibits a variation of $\sim 60 \mathrm{meV}$ at the zoneboundary upon subjecting a thin film of cuprate to a substrateinduced strain. For instance, the spin-wave energy shows hardening near the zone boundary with growing in-plain compressive strain.

In the presence of in-plane strain, the orbital overlap [17, 18] and on-site Coulombic repulsion [18-21] can get affected. The compressive strain enhances the orbital overlap, which results into an increase in the in-plane hopping parameters $(t)$. On the other hand, the separation between the two $e_{g}$ levels also grows, which is expected to push the $d_{3 z^{2}-r^{2}}$-band further below the Fermi level so that the screening of the intra- orbital Coulombic interaction $(U)$ for $d_{x^{2}-y^{2}}$ orbital gets reduced resulting into an increase in $U$. However, $U / t$ may remain constant as suggested by a density-functional theory (DFT) calculation and x-ray absorption spectrum (XAS) measurement [17]. Consequently, the effective exchange coupling $J \approx 4 t^{2} / U$ can increase in the limit of a very large $U$, which has been linked to the hardening of zone-boundary spin-wave energy. Since the separation between the two $e_{g}$ orbitals is directly affected by the in-plane strain, a study based on a model

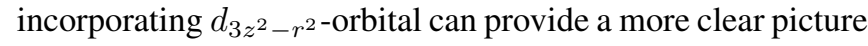
about the origin of variation of zone-boundary spin-wave energy with orbital-splitting (OS), which is undertaken in the current paper.

The importance of $e_{g}$ OS has been emphasized in several recent works including the one which suggested that the difference between the superconducting transition temperature across the high- $T_{c}$ cuprates may depend on the $e_{g}$-level separation. Particularly, the superconducting transition temperature was shown to increase with OS [22-25]. The $e_{g}$ OS $(\delta)$ can range in between $1 \mathrm{eV} \lesssim \delta \lesssim 2 \mathrm{eV}$, whereas the splitting between the two sets $e_{g}$ and $t_{2 g}$ of orbitals is $\approx 2 \mathrm{eV}$ [2, 26$31,40]$. Therefore, while the $d_{x^{2}-y^{2}}$ orbital based one-orbital model can describe the correlation effects for cuprates with a larger $e_{g}$ splitting, it becomes necessary to include both the $e_{g}$ orbitals for the cuprates with a smaller splitting. The evidence from the angle-resolved photoelectron spectroscopy (ARPES) experiments indicates a significant hybridization of bands located not far from the Fermi level, which involves $d_{3 z^{2}-r^{2}}$ orbital [33, 34]. An important role of $d_{3 z^{2}-r^{2}}$ orbital was also indicated in a recent work examining the spin-wave excitations in the hole-doped $\mathrm{La}_{2} \mathrm{CuO}_{4}$ (LSCO) [16]. Presence of this additional orbital may also be responsible for the stability of AFM state against hole doping resulting mainly from the Hund's first rule which demands the maximization of total spin [35].

In this paper, we investigate the role of OS between $d_{x^{2}-y^{2}}$ and $d_{3 z^{2}-r^{2}}$ orbitals in the spin-wave excitations for the AFM phase of the undoped cuprate. In order to achieve this goal, we consider a two-orbital model based on both the $e_{g}$ orbitals. 
Our findings indicate that (i) the zone-boundary spin-wave energy increases with the in-plane compressive strain in the cuprates with a relatively smaller $e_{g}$ splitting such as LSCO, a result in qualitative agreement with recent RIXS experiment. (ii) However, it does not show any significant dependence on strain for the cuprates with a larger splitting. (iii) The behavior originates from the orbital mixing of the lower exchange-split $d_{x^{2}-y^{2}}$ band and a nearly flat $d_{3 z^{2}-r^{2}}$ band. This mixing generates additional exchange coupling based on a second order inter-orbital superexchange process in addition to the intraorbital superexchange.

\section{MODEL}

We consider a Hamiltonian based on the two $e_{g}$ orbitals. The delocalization-energy gain term is given by

$$
\mathcal{H}_{K E}=\sum_{\mathbf{i j}} \sum_{\mu, \nu, \sigma} t_{\mathbf{i} \mathbf{j}}^{\mu \nu} d_{\mathbf{i} \mu \sigma}^{\dagger} d_{\mathbf{j} \nu \sigma} .
$$

$t_{\mathbf{i j}}^{\mu \nu}$ s are the hopping matrix elements from the orbital $\mu$ at site $\mathbf{i}$ to the orbital $\nu$ at site $\mathbf{j}$, respectively. The operator $d_{\mathbf{i} \mu \sigma}^{\dagger}$ $\left(d_{\mathbf{i} \mu \sigma}\right)$ creates (destroys) an electron with spin $\sigma$ at site $\mathbf{i}$ in the orbital $\mu$. The orbitals $\mu$ and $\nu$ are either of the $\mathrm{Cu} 3 d_{x^{2}-y^{2}}$ and $d_{3 z^{2}-r^{2}}$ Wannier orbitals. The $d_{x^{2}-y^{2}}$ Wannier orbital arises due to $3 d_{x^{2}-y^{2}}$ orbital of $\mathrm{Cu}$ and the bridging $2 p_{x / y}$ orbital of $\mathrm{O}$ located in between two $\mathrm{Cu}$ atoms in the $\mathrm{CuO}_{2}$ plane. $d_{3 z^{2}-r^{2}}$ Wannier orbital results from the $\mathrm{Cu} d_{3 z^{2}-r^{2}}$ orbitals and $2 p_{z}$ orbital of $\mathrm{O}$ present in the apical position [23].

The orbital splitting between the $e_{g}$ orbitals $d_{x^{2}-y^{2}}$ and $d_{3 z^{2}-r^{2}}$ is given by

$$
\mathcal{H}_{O S}=\frac{\delta}{2} \sum_{\mathbf{i}}\left(d_{\mathbf{i} \gamma \sigma}^{\dagger} d_{\mathbf{i} \gamma \sigma}-d_{\mathbf{i} \gamma^{\prime} \sigma}^{\dagger} d_{\mathbf{i} \gamma^{\prime} \sigma}\right)
$$

where $\gamma$ and $\gamma^{\prime}$ denote $d_{x^{2}-y^{2}}$ and $d_{3 z^{2}-r^{2}}$ orbitals, respectively. $\delta$ is the orbital splitting parameter, which is controlled by the distance of the apical oxygen from the $\mathrm{CuO}_{2}$ plane. The in-plane strain applied on a thin film can generate a modification in both in-plane and out-of-plane lattice parameter, which can introduce a change in the overall crystal-field effect. Consequently, $\delta$ gets directly affected. The same has been indicated by the XAS and RIXS measurements. The $d d$ excitations study based on the $\mathrm{Cu} L_{3}$ edge, shows that the center of mass of $d d$ excitations shift systematically towards higher energy with increasing in-plane compressive strain. The dependence of the position of the center of mass on the strain parameter defined as $\epsilon=\left(a-a_{0}\right) / a_{0}$ is nearly linear. This may also imply a similar enhancement in the $e_{g}$ orbital splittings as a function of $\epsilon$ [17].

The standard on-site Coulomb interaction is given by

$$
\begin{aligned}
\mathcal{H}_{i n t} & =U \sum_{\mathbf{i}, \mu} n_{\mathbf{i} \mu \uparrow} n_{\mathbf{i} \mu \downarrow}+\left(U^{\prime}-\frac{J}{2}\right) \sum_{\mathbf{i}} n_{\mathbf{i} \gamma} n_{\mathbf{i} \gamma^{\prime}} \\
& -2 J \sum_{\mathbf{i}} \mathbf{S}_{\mathbf{i} \gamma} \cdot \mathbf{S}_{\mathbf{i} \gamma^{\prime}}+J \sum_{\mathbf{i}, \sigma} d_{\mathbf{i} \gamma \sigma}^{\dagger} d_{\mathbf{i} \gamma \bar{\sigma}}^{\dagger} d_{\mathbf{i} \gamma^{\prime} \bar{\sigma}} d_{\mathbf{i} \gamma^{\prime} \sigma} .
\end{aligned}
$$
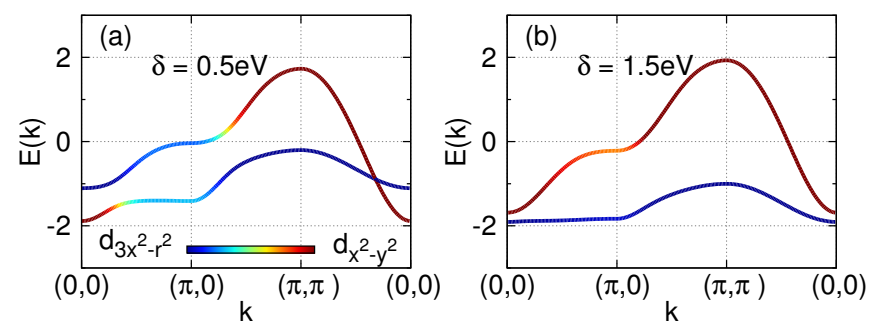

FIG. 1: The orbital contents of electronic bands along the highsymmetry directions in the two-orbital model when the orbital splittings $(\delta$ ) are (a) $0.5 \mathrm{eV}$ and (b) $1.5 \mathrm{eV}$. The orbital mixing is dominant near $(\pi, 0)$.

The intra- and inter-orbital Coulomb interaction $\left(U\right.$ and $\left.U^{\prime}\right)$ terms are described by the first and second terms, respectively, where $n_{\mathbf{i} \mu \sigma}=d_{\mathbf{i} \mu \sigma}^{\dagger} d_{\mathbf{i} \mu \sigma}$ and $n_{\mathbf{i} \gamma}=\sum_{\sigma} d_{\mathbf{i} \gamma \sigma}^{\dagger} d_{\mathbf{i} \gamma \sigma}$ with $\sigma=\uparrow, \downarrow$. The third term stands for the Hund's coupling between electrons of different orbitals, where $S_{\mathbf{i} \gamma}^{l}=$ $\sum_{\sigma \sigma^{\prime}} d_{\mathbf{i} \gamma \sigma}^{\dagger} \sigma_{\sigma \sigma^{\prime}}^{l} d_{\mathbf{i} \gamma \sigma} . \sigma^{l} s$ are the Pauli matrices with $l=$ $x, y, z$. The last term is the pair-hopping term.

\section{METHOD}

The mean-field decoupling of various interaction terms in Eq. 1 originating from the Coulombic interaction yields the following mean-field Hamiltonian [36]

$$
H_{\mathbf{k}}=\sum_{\mathbf{k} \sigma} \Psi_{\mathbf{k} \sigma}^{\dagger}\left[\begin{array}{cc}
\hat{h}(\mathbf{k})+\hat{N} & \operatorname{sgn} \bar{\sigma} \hat{M} \\
\operatorname{sgn} \bar{\sigma} \hat{M} & \hat{h}(\mathbf{k}+\mathbf{Q})+\hat{N}
\end{array}\right] \Psi_{\mathbf{k} \sigma}
$$

for the $(\pi, \pi)$ AFM state in the momentum space. $\Psi_{\mathbf{k} \sigma}^{\dagger}=$ $\left(d_{\mathbf{k} 1 \sigma}^{\dagger}, d_{\mathbf{k} 2 \sigma}^{\dagger}, d_{\mathbf{k} \overline{1} \sigma}^{\dagger}, d_{\mathbf{k} \overline{2} \sigma}^{\dagger}\right)$ with $d_{\mathbf{k} \bar{l} \sigma}^{\dagger}=d_{\mathbf{k}+\mathbf{Q} l \sigma}^{\dagger}$ and $\mathbf{Q}=$ $(\pi, \pi)$. The elements of the $2 \times 2$ matrix $\hat{h}_{\mathbf{k}}$ are given by

$$
\begin{aligned}
h_{11}(\mathbf{k}) & =-2 t_{1}\left(\cos k_{x}+\cos k_{y}\right)+4 t_{2} \cos k_{x} \cos k_{y} \\
& -2 t_{3}\left(\cos 2 k_{x}+\cos 2 k_{y}\right) \\
h_{12}(\mathbf{k}) & =h_{21}(\mathbf{k})=2 t_{4}\left(\cos k_{x}-\cos k_{y}\right) \\
& +2 t_{5}\left(\cos 2 k_{x}-\cos 2 k_{y}\right) \\
h_{22}(\mathbf{k}) & =-2 t_{6}\left(\cos k_{x}+\cos k_{y}\right),
\end{aligned}
$$

where the hopping parameters are $t_{1}=0.452, t_{2}=0.0895$, $t_{3}=0.0705, t_{4}=0.171, t_{5}=0.0248, t_{6}=0.113$ with the unit being eV. $t_{1}, t_{2}$ and $t_{3}$ are the nearest, next-nearest and next-next-nearest neighbor intra-orbital hopping parameters for the $d_{x^{2}-y^{2}}$ orbitals. $t_{4}$ and $t_{5}$ are the nearest and next-next-nearest neighbor inter-orbital hopping parameters. $t_{6}$ is the nearest neighbor intra-orbital hopping parameter for the $d_{3 z^{2}-r^{2}}$ orbitals.

$\hat{M}$ and $\hat{N}$ are $2 \times 2$ matrices with the elements given in terms of the interaction parameters, charge densities and magnetization. $2 M_{l l}=U m_{l l}+J \sum_{l \neq m} m_{m m}$ and $2 M_{l m}=$ $J m_{l m}+(U-2 J) m_{m l}$. Also, $2 N_{l l}=U n_{l l}+(2 U-$ $5 J) \sum_{l \neq m} n_{m m}$ and $2 N_{l m}=J n_{l m}+(4 J-U) n_{m l}$. The self-consistent mean-field order parameters, i.e. charge density and magnetization are given by $n_{\mu \nu}=\sum_{\mathbf{k} \sigma}\left\langle d_{\mathbf{k} \mu \sigma}^{\dagger} d_{\mathbf{k} \nu \sigma}\right\rangle$ and $m_{\mu \nu}=\sum_{\mathbf{k} \sigma}\left\langle d_{\mathbf{k}+\mathbf{Q} \mu \sigma}^{\dagger} d_{\mathbf{k} \nu \sigma}\right\rangle \operatorname{sgn} \sigma$. 

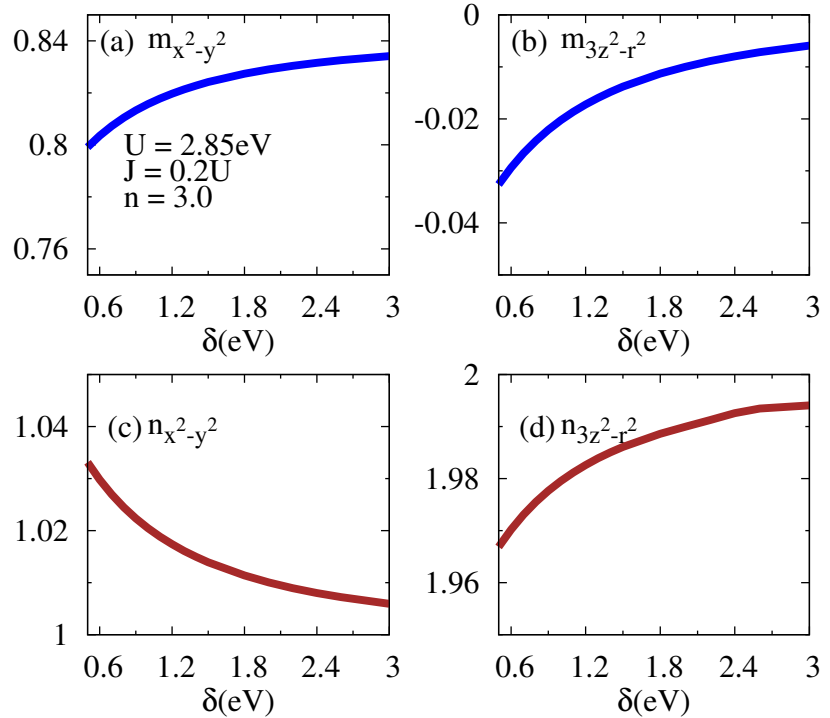

FIG. 2: Magnetizations for the orbitals (a) $d_{x^{2}-y^{2}}$ and (b) $d_{3 z^{2}-r^{2}}$ show opposite behavior as a function of the OS. It increases for the former while decreases in magnitude for the latter when the OS is increased. Note that a negative $m_{3 z^{2}-r^{2}}$ indicates that the magnetization is oriented in a direction opposite to $m_{x^{2}-y^{2}}$. At the same time, the orbital-resolved charge density (a) $n_{x^{2}-y^{2}}$ decreases and (b) $n_{3 z^{2}-r^{2}}$ increases.

In order to study the spin-wave excitations in the AFM state, we calculate the transverse spin susceptibility

$$
\begin{array}{r}
\chi_{\alpha \beta, \mu \nu}^{+-}\left(\mathbf{q}, \mathbf{q}^{\prime}, i \omega_{n}\right)= \\
T \int_{0}^{1 / T} d \tau e^{i \omega_{n} \tau}\left\langle T_{\xi}\left[S_{\alpha \beta}^{+}(\mathbf{q}, \tau) S_{\nu \mu}^{-}\left(-\mathbf{q}^{\prime}, 0\right)\right]\right\rangle
\end{array}
$$

within the two-orbital model. Here, $\mathbf{q}, \mathbf{q}^{\prime}=\mathbf{q}$ or $\mathbf{q}+$ Q. The components of the spin operators are given by $S_{\alpha \beta}^{i}(\mathbf{q})=\sum_{\mathbf{k}} \sum_{\sigma \sigma^{\prime}} d_{\alpha \sigma}^{\dagger}(\mathbf{k}+\mathbf{q}) \sigma_{\sigma \sigma^{\prime}}^{i} d_{\beta \sigma^{\prime}}(\mathbf{k}) . \sigma^{i}$ are Pauli matrices while the subscripts $\sigma, \sigma^{\prime}=\uparrow, \downarrow$. Using the meanfield Hamiltonian described by Eq. 4, the bare Green's functions $G_{\alpha \mu}^{\uparrow}\left(\mathbf{k}, i \omega_{n}\right)$ can be obtained. The transverse-spin susceptibility for the AFM state in the random-phase approximation is calculated as

$$
\hat{\bar{\chi}}\left(\mathbf{q}, i \omega_{n}\right)=\left(\hat{\mathbf{1}}-\hat{\chi}\left(\mathbf{q}, i \omega_{n}\right) \hat{U}\right)^{-1} \hat{\chi}\left(\mathbf{q}, i \omega_{n}\right)
$$

where $\hat{\mathbf{1}}$ is a $8 \times 8$ identity matrix and $\hat{U}$ is a blockdiagonal interaction matrix with the elements of both the blocks being identical. $\hat{\bar{\chi}}\left(\mathbf{q}, i \omega_{n}\right)$ and $\hat{\chi}\left(\mathbf{q}, i \omega_{n}\right)$ are $8 \times$ 8 matrices, which is evident from the structure of Eq. 6 . Note that each element of the susceptibility matrix $\hat{\chi}\left(\mathbf{q}, i \omega_{n}\right)$ contains $\chi_{\alpha \beta, \mu \nu}\left(\mathbf{q}, \mathbf{q}, i \omega_{n}\right)=\sum_{\mathbf{k}, i \omega_{n}^{\prime}} G_{\alpha \mu}^{\uparrow}\left(\mathbf{k}+\mathbf{q}, i \omega_{n}^{\prime}+\right.$ $\left.i \omega_{n}\right) G_{\nu \beta}^{\downarrow}\left(\mathbf{k}, i \omega_{n}^{\prime}\right)$ when $\mathbf{q}^{\prime}=\mathbf{q}$ as well as the terms arising due to the Umklapp processes. The physical spin susceptibility using the appropriate elements of the $\hat{\bar{\chi}}\left(\mathbf{q}, i \omega_{n}\right)$ is given by $\bar{\chi}_{p h}\left(\mathbf{q}, i \omega_{n}\right)=\sum_{\alpha \mu} \bar{\chi}_{\alpha \alpha, \mu \mu}\left(\mathbf{q}, \mathbf{q}, i \omega_{n}\right)[37]$.
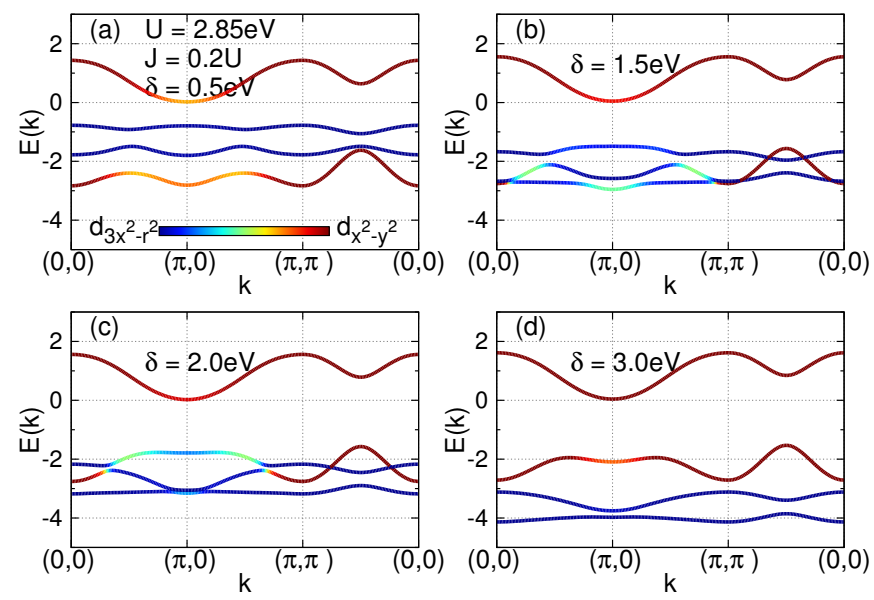

FIG. 3: Predominant orbitals in all the four reconstructed bands of the AFM state along the high-symmetry directions for $\delta=$ (a) $0.5 \mathrm{eV}$, (b) $1.5 \mathrm{eV}$, (c) $2.0 \mathrm{eV}$ and (d) $3.0 \mathrm{eV}$. A significant orbital mixing in the lower exchange-split band and $d_{3 z^{2}-r^{2}}$ dominated band is present for $\delta \approx 2 \mathrm{eV}$.

\section{RESULTS AND DISCUSSION}

Fig. 1 shows the electronic dispersions in the two-orbital model for the OS (a) $\delta=0.5 \mathrm{eV}$ and (b) $1.5 \mathrm{eV}$. There is no mixing of $d_{x^{2}-y^{2}}$ and $d_{3 z^{2}-r^{2}}$ orbitals in the bands along the $(0,0)-(\pi, \pi)$ direction as $h_{12}(\mathbf{k})$ vanishes identically. The mixing is maximum near $(\pi, 0)$ in the vicinity of Van Hove singularity because $h_{12}(\mathbf{k})$ attains it's maximum value at the same point. In other directions, the orbital mixing is moderate. As the OS increases, the two bands are increasingly orbitally polarized and they become almost completely polarized for $\delta \approx 1.5 \mathrm{eV}$ and beyond. In the limit of very small $e_{g}$ splitting the bands will resemble to that of the monolayer manganites [38].

Fig. 2 shows the charge densities and magnetic order parameters for the orbitals $d_{x^{2}-y^{2}}$ and $d_{3 z^{2}-r^{2}}$ as a function of OS in the AFM state. The total charge density $n=3.0$ is fixed throughout the paper unless stated otherwise, which corresponds to the scenario with nearly half-filled $d_{x^{2}-y^{2}}$ orbital and completely filled $d_{3 z^{2}-r^{2}}$ orbital. The charge density $n_{x^{2}-y^{2}}$ in the $d_{x^{2}-y^{2}}$ orbital decreases while $n_{3 z^{2}-r^{2}}$ increases as the OS increases, which results from the constraint that the total charge density is fixed while the electrons will occupy the low-energy states first. However, $n_{x^{2}-y^{2}}>1$ by $\approx 2 \%$ even if $\delta \approx 1.5 \mathrm{eV}$ a value greater than what is considered widely acceptable for LSCO. Thus, the $d_{3 z^{2}-r^{2}}$ orbital is not completely filled and therefore can play an important role in the spin-wave excitations to be discussed below. The magnetization $m_{x^{2}-y^{2}}$ in the $d_{x^{2}-y^{2}}$ orbital increases continuously with a rise in the OS. This is mainly a consequence of the fact that the double occupancy diminishes as the OS increases. On the other hand, the magnitude of $m_{3 z^{2}-r^{2}}$ drops as the double occupancy increases.

Fig. 3 shows the reconstructed band in the AFM state for various OSs. Unlike the $d_{x^{2}-y^{2}}$ band, the exchange splitting 
for $d_{3 z^{2}-r^{2}}$ dominated band is small because $d_{3 z^{2}-r^{2}}$ orbitals are nearly doubly occupied. For $\delta=0.5 \mathrm{eV}$, the $d_{3 z^{2}-r^{2}}$ dominated bands are located in between the exchange-split $d_{x^{2}-y^{2}}$ bands. Moreover, the mixing of orbitals in the bands are minimal. But the separation between the relatively narrow $d_{3 z^{2}-r^{2}}$ band and the lower-exchange split $d_{x^{2}-y^{2}}$ band decreases with the rise in OS. This results in an increased mixing of the two orbitals in the three low lying bands. With further rise in the OS, the orbital mixing maximizes and thereafter it decreases so that there are almost completely polarized two upper bands dominated by the $d_{x^{2}-y^{2}}$ orbital and two lower bands dominated by the $d_{3 z^{2}-r^{2}}$ orbital as shown in Fig. 3(d).

Fig. 4 shows the spin-wave excitation energy calculated by using imaginary part of $\bar{\chi}_{p h}\left(\mathbf{q}, i \omega_{n}\right)$ as a function of $\delta$ along the high-symmetry direction. We have chosen the intra-orbital Coulomb interaction parameter $U=2.85 \mathrm{eV}$, Hund's coupling $J=0.2 U$ and $\delta \approx 1 \mathrm{eV}$ so that the spin-wave excitations shows a good agreement with the neutron-scattering experiments for LSCO. It is worthwhile to note that there may a non-negligible magnon-self energy correction due to coupling of spin degree of freedom with charge and orbital degree of freedom [42].

Earlier, the necessity of a similar range of $U$ was stressed in the one-orbital model for different cuprates [8, 13, 14]. The estimates by studies based on the LDA + DMFT or the photoemission spectroscopy also yields a similar value of $U$ $\sim 3 \mathrm{eV}[40,41]$. For this range of on-site Coulomb interaction parameter, the magnetization $m_{x^{2}-y^{2}} \sim 0.9$ in a selfconsistent meanfield theory, which is significantly larger than the experimental estimates $0.55 \mu_{B}$. However, by going beyond the meanfield level, it can be shown that the correction to the meanfield sublattice magnetization originating due to the spin fluctuations may yield a reduction up to $40 \%$ [43]. This brings the sublattice magnetization to a value very close to what is observed experimentally. It is true that the corrections to the sublattice magnetization were obtained only within the one-orbital model, it is though not unreasonable to expect that the magnitude of correction will be of a similar order even in the two-orbital model.

More importantly, the zone-boundary excitations show a significant dependence on the OS. In particular, we find that the zone-boundary spin-wave energy increases with the OS within the range $0.5 \mathrm{eV} \lesssim \delta \lesssim 2.0 \mathrm{eV}$. The growth is monotonic at the high symmetry point $(1 / 2,0)$. Beyond $\delta \sim 2.0 \mathrm{eV}$, the zone-boundary spin-wave energy starts decreasing but the rate of decline is comparatively smaller than the rate of rise noted for $\delta \lesssim 2.0 \mathrm{eV}$.

The hardening of zone-boundary spin-wave energy also implies an enhancement in the effective exchange coupling, which in turn may indicate a more stable AFM state. The stabilization may result from the presence of an additional channel for lowering of energy, which is specific to the two-orbital models. While in the one-orbital model, there is only a single channel for the second order super-exchange interaction, several channels for the second order exchange interactions are possible in the two-orbital models.

The zone-boundary hardening of the spin-wave excitation energy occurs because of the virtual process involving the

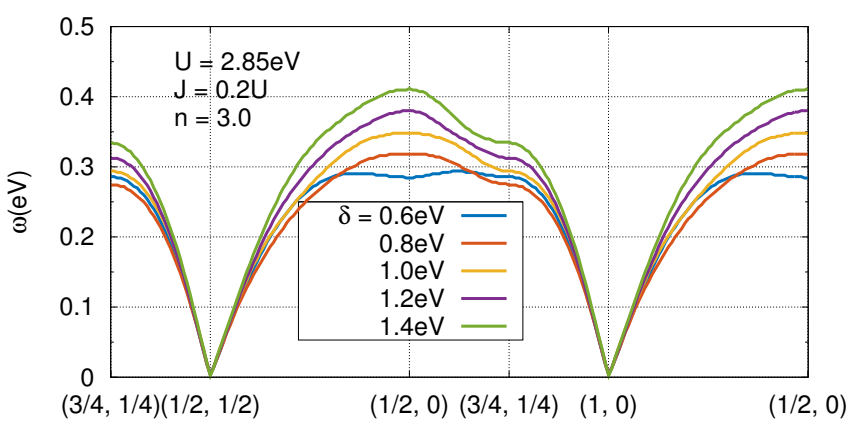

q

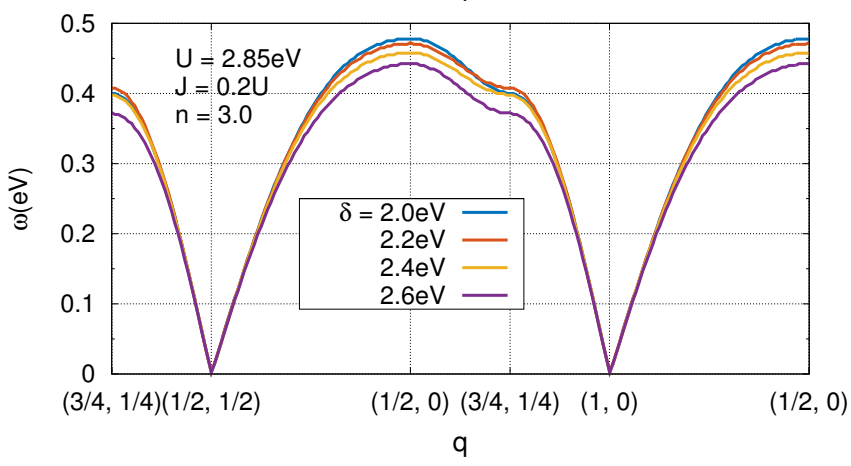

FIG. 4: Spin-wave excitations along the high-symmetry directions. Zone-boundary spin-wave energy shows a significant dependence on the orbital splitting between $d_{x^{2}-y^{2}}$ and $d_{3 z^{2}-r^{2}}$ orbitals within the range $0.5 \mathrm{eV} \lesssim \delta \lesssim 2.0 \mathrm{eV}$, while there is only a weaker dependence beyond $\delta=2.0 \mathrm{eV}$.

hopping of a $d_{3 z^{2}-r^{2}}$ electron to a neighboring $d_{x^{2}-y^{2}}$ orbital and then returning back, which is possible as the inter-orbital hopping is non zero. More specifically, when the magnetic moments are considered oriented along the $z$-direction as in the current paper, an additional antiferromagnetic exchange coupling can be generated because an $\uparrow$-spin $d_{3 z^{2}-r^{2}}$ electron from a site with $\uparrow$-spin $d_{x^{2}-y^{2}}$ electron can hop to the $d_{x^{2}-y^{2}}$ orbital at a nearest-neighbor site occupied already by a $\downarrow$-spin electron and then return back to it's original position.

The energy of the $\uparrow$-spin $d_{3 z^{2}-r^{2}}$ electron at it's original site is $\approx U+U^{\prime}-J$. The $d_{3 z^{2}-r^{2}}$ orbital is doubly occupied therefore there is a contribution of $U$ due to the intra-orbital Coulomb interaction. Similarly, there is also a contribution of $U^{\prime}$ due to the inter-orbital Coulomb interaction. Further, the energy is lowered by $J$ because of the Hund's coupling between the $\uparrow$-spin $d_{3 z^{2}-r^{2}}$ and $d_{x^{2}-y^{2}}$ electrons at the original site.

When the $\uparrow$-spin $d_{3 z^{2}-r^{2}}$ electron transfers to the $d_{x^{2}-y^{2}}$ orbital at the neighboring site, it's Coulombic energies are $U$ and $2 U^{\prime}$ due to the intra- and inter-orbital interactions, respectively. There is no contribution from the Hund's coupling term because $d_{3 z^{2}-r^{2}}$ is almost doubly occupied. Thus, the total Coulombic energy at the new site is $\approx U+2 U^{\prime}+\delta$, where we have also incorporated the fact that $d_{x^{2}-y^{2}}$ and $d_{3 z^{2}-r^{2}}$ orbitals are separated by the energy $\delta$ because of the orbital splitting.

Such a process leads to the antiferromagnetic exchange coupling $J_{i n t} \approx-4 t_{4}^{2} /\left(\delta+U^{\prime}+J\right)=-4 t_{4}^{2} /(\delta+U-J)$, 


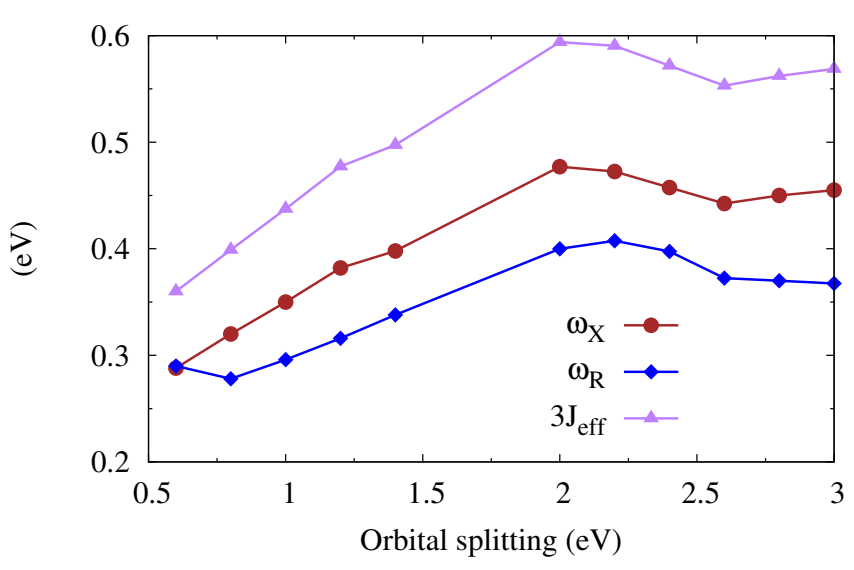

FIG. 5: The zone-boundary spin-wave excitation energies and the effective exchange coupling as a function of orbital splitting between the $d_{x^{2}-y^{2}}$ and $d_{3 z^{2}-r^{2}}$ orbitals.

where $t_{4}$ is the inter-orbital hopping parameter and $U=$ $U^{\prime}+2 J$ in accordance with the rotational symmetry of the Hamiltonian. The contribution of this term is negligible when the lower exchange-split $d_{x^{2}-y^{2}}$ band and $d_{3 z^{2}-r^{2}}$ band don't mix much as is the case when the OS is either very small ( $\sim$ $0.5 \mathrm{eV})$ or too large $(\sim 3.0 \mathrm{eV})$. However, at the intermediate value of $\delta \sim 2 \mathrm{eV}$, the contribution is significant as the orbital mixing is non-negligible. Note the presence of $\delta$ in the denominator of $J_{i n t}$, which explains a faster rise in the zoneboundary spin-wave energy for a smaller OS within the range $0.5 \mathrm{eV} \lesssim \delta \lesssim 2.0 \mathrm{eV}$ and a slower decline beyond $\delta \sim 2.0 \mathrm{eV}$ for a larger OS.

Fig. 5 shows the zone-boundary spin-wave excitations $\omega$ at $\mathrm{X} \equiv(1 / 2,0)$ and $\mathrm{R} \equiv(3 / 4,1 / 4)$ as a function of $\delta$. Both show almost a linear growth up to $\delta \approx 2.0 \mathrm{eV}$, thereafter they don't show much variation. The region with a nearly linear dependence shows a very good qualitative agreement with the observed zone-boundary spin-wave excitations in the samples subjected to the in-plane strain. We have also plotted the effective-exchange coupling determined from the approximate relation $J_{\text {eff }}=\omega_{X} / 2 Z$, where the renormalization factor resulting from the self-energy correction is $Z=$ $1.2[14,16]$. As the exchange coupling displays a nearly linear dependence on the in-plane compressive strain, our finding suggests that the $e_{g}$ orbital splitting $\delta$ may also exhibit a nearly linear dependence on the in-plane strain.

\section{SUMMARY AND CONCLUSIONS}

The spin-wave excitations in the cuprates has been largely explored within a one-orbital model. In a recent work, the two-orbital model was invoked to explain the difference between the superconducting-transition temperature across different cuprates using the fluctuation-exchange approximation which incorporates the spin-spin correlations [22-25]. An in- direct implication of the above result is that the spin-wave excitations in different phases including the AFM may also show dependence on the OS induced by the in-plain stress.

The strain in the layered cuprates affects not only the overlap integral between the orbitals at neighboring sites but it can also lead to a non negligible modification in the on site Coulombic interaction. The net possible impact of the interplay between the aforementioned consequences on the spinwave excitations is yet to be fully understood. However, the most significant impact of in-plain strain perhaps is on the extent of orbital mixing for the bands either located near or far from the Fermi surface. As illustrated through the current work, even if we ignore the modification in overlap integral and Coulomb interaction, the two-orbital model successfully describes the experimental observations in terms of orbital mixing present in various bands.

Our study is focused at zero doping where the cuprates show only the antiferromagnetic order. On doping holes, the long-range magnetic order is lost. However, the nature of leading order local magnetic-exchange couplings are expected to show a weak dependence on doping. The higher spin-wave excitation energy for a larger $\delta$ may indicate an enhancement in the exchange coupling which will remain true even on doping holes. As found, the spin-wave excitation energy increases with $\delta$ for a realistic range so does the magnetic-exchange coupling. Therefore, the high energy spin-fluctuations would help to increase the superconducting transition temperature $T_{c}$. However, this is true only for those cuprates for which $\delta \lesssim 2 \mathrm{eV}$. The cuprates such as $\mathrm{HgBa}_{2} \mathrm{CuO}_{4}$ has a relatively larger $\delta \gtrsim 2 \mathrm{eV}$. According to our calculation, the spin-wave excitation energy does not increase on increasing $\delta$ near $2 \mathrm{eV}$. Thus, by applying in-plane compressive strain we may not be able to increase $T_{C}$ of $\mathrm{HgBa}_{2} \mathrm{CuO}_{4}$ except for the lower $e_{g}$ split cuprates such as $\mathrm{La}_{2} \mathrm{CuO}_{4}$ [22-24].

In summary, we have explored the spin-wave excitations in the undoped AFM state of cuprates within a two-orbital model based on $d_{x^{2}-y^{2}}$ and $d_{3 z^{2}-r^{2}}$ orbitals. Our investigation reveals that the zone-boundary spin-wave energy hardens with an increase in the orbital splitting for the range $0.5 \mathrm{eV} \lesssim \delta \lesssim$ $2.0 \mathrm{eV}$. The result, besides providing a plausible explanation for the recent observations in RIXS measurements, emphasizes also on the importance of $d_{3 z^{2}-r^{2}}$ orbital in the cuprates with smaller $e_{g}$ splitting.

\section{Acknowledgement}

We would like to thank the anonymous referee for bringing our attention to the issue explored in this work. We acknowledge the use of HPC clusters at HRI. D. K. Singh was supported through start-up grant SRG/2020/002144 funded by DST-SERB. Y. Bang was supported through NRF Grant No. 2020-R1A2C2-007930 funded by the National Research Foundation of Korea. 
[1] J. G. Bednorz and K. A. Müller, Zeitschrift für, Physik B Condensed Matter 64, 189 (1986).

[2] M. Imada, A. Fujimori, and Y. Tokura, Rev. Mod. Phys. 70, 1039 (1998).

[3] A. Damascelli, Z. Hussain, and Z.-X. Shen, Rev. Mod. Phys. 75, 473 (2003).

[4] P. A. Lee, N. Nagaosa, and X.-G. Wen, Rev. Mod. Phys. 78, 17 (2006).

[5] Y. Kamihara, T. Watanabe, M. Hirano, H. Hosono, J. Am. Chem. Soc. 130, 3296 (2008).

[6] L. Boeri, O. V. Dolgov, and A. A. Golubov Phys. Rev. Lett. 101, 026403 (2008).

[7] Q. Si, R. Yu, and E. Abrahams, Nature Rev. Mater. 1, 16017 (2016).

[8] R. Coldea, S. M. Hayden, G. Aeppli, T. G. Perring, C. D. Frost, T. E. Mason, S.-W. Cheong, and Z. Fisk, Phys. Rev. Lett. 86, 5377 (2001).

[9] L. Braicovich, L. J. P. Ament, V. Bisogni, F. Forte, C. Aruta, G. Balestrino, N. B. Brookes, G. M. De Luca, P. G. Medaglia, F. Miletto Granozio, M. Radovic, M. Salluzzo, J. van den Brink, and G. Ghiringhelli, Phys. Rev. Lett. 102, 167401 (2009).

[10] N. S. Headings, S. M. Hayden, R. Coldea, and T. G. Perring Phys. Rev. Lett. 105, 247001 (2010).

[11] M. P. M. Dean, R. S. Springell, C. Monney, K. J. Zhou, J. Pereiro, I. Božović, B. Dalla Piazza, H. M. Rønnow, E. Morenzoni, J. van den Brink, T. Schmitt and J. P. Hill, Nat. Mat. 11, 850 (2012).

[12] Y. Peng, G. Dellea, M. Minola, M. Conni, A. Amorese, D. Di Castro, G. M. De Luca, K. Kummer, M. Salluzzo, X. Sun, X. J. Zhou, G. Balestrino, M. Le Tacon, B. Keimer, L. Braicovich, N. B. Brookes, and G. Ghiringhelli, Nat. Phys. 13, 1201 (2017).

[13] A. Singh and P. Goswami, Phys. Rev. B 66, 092402 (2002).

[14] J.-Y. P. Delannoy, M. J. P. Gingras, P. C. W. Holdsworth, and A.-M. S. Tremblay, Phys. Rev. B 79, 235130 (2009).

[15] J. M. P. Carmelo, M. A. N. Araújo, S. R. White, and M. J. Sampaio Phys. Rev. B 86, 064520 (2012).

[16] O. Ivashko, N. E. Shaik, X. Lu, C. G. Fatuzzo, M. Dantz, P. G. Freeman, D. E. McNally, D. Destraz, N. B. Christensen, T. Kurosawa, N. Momono, M. Oda, C. E. Matt, C. Monney, H. M. Ronnow, T. Schmitt, and J. Chang, Phys. Rev. B 95, 214508 (2017).

[17] O. Ivashko, M. Horio, W. Wan, N. B. Christensen, D. E. McNally, E. Paris, Y. Tseng, N. E. Shaik, H. M. Ronnow, H. I. Wei, C. Adamo, C. Lichtensteiger, M. Gibert, M. R. Beasley, K. M. Shen, J. M. Tomczak, T. Schmitt and J. Chang, Nat. Comm. 10, 786 (2019).

[18] M. Abrecht, D. Ariosa, D. Clotta, S. Mitrovic, M. Onellion, X. X. Xi, G. Margaritondo, and D. Pavuna, Phys. Rev. Lett. 91, 057002 (2003).

[19] Jan M. Tomczak, T. Miyake, R. Sakuma, and F. Aryasetiawan, Phys. Rev. B 79, 235133 (2009).

[20] Jan M. Tomczak, T. Miyake, and F. Aryasetiawan, Phys. Rev. B 81, 115116 (2010).

[21] B. Kim, P. Liu, Jan M. Tomczak, and C. Franchini, Phys. Rev. B 98, 075130 (2018).

[22] H. Sakakibara, H. Usui, K. Kuroki, R. Arita, and H. Aoki, Phys. Rev. Lett. 105, 057003 (2010).

[23] H. Sakakibara, H. Usui, K. Kuroki, R. Arita, and H. Aoki, Phys.
Rev. B 85, 064501 (2012).

[24] H. Sakakibara, K. Suzuki, H. Usui, S. Miyao, I. Maruyama, K. Kusakabe, R. Arita, H. Aoki and K. Kuroki, Phys. Rev. B 89, 224505 (2014).

[25] H. B. Tang, G. Li, H. J. Zhang, X. Q. Zuo, F. M. Meng, and D. Y. Liu, Physica B 46845 (2015).

[26] J. Lorenzana and G. A. Sawatzky, Phys. Rev. Lett. 74, 1867 (1995).

[27] C. de Graaf and R. Broer, Phys. Rev. B 62, 702 (2000).

[28] J. P. Hill, G. Blumberg, Y.-J. Kim, D. S. Ellis, S. Wakimoto, R. J. Birgeneau, S. Komiya, Y. Ando, B. Liang, R. L. Greene, D. Casa, and T. Gog, Phys. Rev. Lett. 100, 097001 (2008).

[29] M. M. Sala, V. Bisogni, C. Aruta, G. Balestrino, H. Berger, N. B. Brookes, G. M. de Luca, D. D. Castro, M. Grioni, M. Guarise, P. G. Medaglia, F. M. Granozio, M. Minola, P. Perna, M. Radovic, M. Salluzzo, T. Schmitt, K. J. Zhou, L. Braicovich, and G. Ghiringhelli, New Journal of Physics 13, 043026 (2011).

[30] G. Ghiringhelli, N. B. Brookes, E. Annese, H. Berger, C. Dallera, M. Grioni, L. Perfetti, A. Tagliaferri, and L. Braicovich, Phys. Rev. Lett. 92, 117406 (2004).

[31] L. Hozoi, L. Siurakshina, P. Fulde, and J. van den Brink, Sci. Rep. 1, 65 (2011).

[32] S. W. Jang, T. Kotani, H. Kino, K. Kuroki, and M. J. Han, Sci. Rep. 5, 12050 (2015).

[33] C. E. Matt, D. Sutter, A. M. Cook, Y. Sassa, M. Mransson, O. Tjernberg, L. Das, M. Horio, D. Destraz, C. G. Fatuzzo, K. Hauser, M. Shi, M. Kobayashi, V. N. Strocov, T. Schmitt, P. Dudin, M. Hoesch, S. Pyon, T. Takayama, H. Takagi, O. J. Lipscombe, S. M. Hayden, T. Kurosawa, N. Momono, M. Oda, T. Neupert, and J. Chang, Nat. Comm. 9, 972 (2018).

[34] K. P. Kramer, M. Horio, S. S. Tsirkin, Y. Sassa, K. Hauser, C. E. Matt, D. Sutter, A. Chikina, N. B. M. Schröter, J. A. Krieger, T. Schmitt, V. N. Strocov, N. C. Plumb, M. Shi, S. Pyon, T. Takayama, H. Takagi, T. Adachi, T. Ohgi, T. Kawamata, Y. Koike, T. Kondo, O. J. Lipscombe, S. M. Hayden, M. Ishikado, H. Eisaki, T. Neupert, and J. Chang, Phys. Rev. B 99, 224509 (2019).

[35] D. K. Singh, A. Go, H.-Y. Choi, and Y. Bang, New J. Phys. 22 063048 (2020).

[36] M. Kovacic, M. H. Christensen, M. N. Gastiasoro, and B. M. Andersen, Phys. Rev. B 91, 064424 (2015).

[37] J. Knolle, I. Eremin, and R. Moessner, Phys. Rev. B 83, 224503 (2011); D. K. Singh, Journal of Applied Physics 122, 073906 (2017), D. K. Singh, J. Phys.: Condens. Matter 29415601 (2017).

[38] D. K. Singh, Eur. Phys. J. B 88, 101 (2015).

[39] A. Singh and P. Goswami, Phys. Rev. B 66, 092402 (2002).

[40] S. W. Jang, H. Sakakibara, H. Kino, T. Kotani, K. Kuroki and M. J. Han, Sci Rep 6, 33397 (2016).

[41] F. Nilsson, K. Karlsson, and F. Aryasetiawan, Phys. Rev. B 99, 075135 (2019).

[42] M. Snamina and A. M Olé, New J. Phys. 21023018 (2019); S. Pandey and A. Singh, Phys. Rev. B 78, 014414 (2008); D. K. Singh, B. Kamble, and A. Singh Phys. Rev. B 81, 064430 (2010)

[43] A. Singh and Z. Tešanović, Phys. Rev. B 41, 614 (1990). 